\title{
Glucose and Fat Oxidation: Bomb Calorimeter Be Damned
}

\author{
Christopher B. Scott \\ Department of Exercise, Health, and Sport Sciences, University of Southern Maine, 37 College Avenue, \\ Gorham, ME 04038, USA \\ Correspondence should be addressed to Christopher B. Scott, cscott@usm.maine.edu
}

Received 7 October 2011; Accepted 14 November 2011

Academic Editors: K. M. Botham and A. Chicco

Copyright (C) 2012 Christopher B. Scott. This is an open access article distributed under the Creative Commons Attribution License, which permits unrestricted use, distribution, and reproduction in any medium, provided the original work is properly cited.

\begin{abstract}
For both respiration and combustion, the energy loss difference between glucose and fat oxidation often is referenced to the efficiency of the fuel. Yet, the addition of anaerobic metabolism with ATP resynthesis to complete respiratory glucose oxidation further contributes to energy loss in the form of entropy changes that are not measured or quantified by calorimetry; combustion and respiratory fat/lactate oxidation lack this anaerobic component. Indeed, the presence or absence of an anaerobic energy expenditure component needs to be applied to the estimation of energy costs in regard to glucose, lactate, and fuel oxidation, especially when the measurement of oxygen uptake alone may incorrectly define energy expenditure.
\end{abstract}

\section{Introduction}

Antoine Lavoisier (1741-1794) provided a brilliant start. "La respiration est donc une combustion" he mused, well before thermodynamic distinctions were refined by acknowledging the type of fuel undergoing oxidation and almost 150 years prior to the founding of metabolic biochemistry: with glucose oxidation, 1 liter of $\mathrm{O}_{2}$ uptake $\approx 21.1 \mathrm{~kJ}$; with fat oxidation, 1 liter of $\mathrm{O}_{2}$ uptake $\approx 19.6 \mathrm{~kJ}$. From this description, the $1.5 \mathrm{~kJ}$ difference between substrates would appear to influence $\mathrm{O}_{2}$ consumption based on cellular energy demands [1]. As an example, $1000 \mathrm{~kJ}$ of energy would require the consumption of 47.4 liters of $\mathrm{O}_{2}$; with fat as a fuel, 51.0 liters are consumed. Yet, in some cases, substrate distinction is no longer considered because the measurement of $\mathrm{O}_{2}$ uptake in and of itself has been used to "quantify" energy costs. Direct measurements of heat loss-kJoules-are now rarely collected or reported. As this paper demonstrates, there is more than meets the eye in an $\mathrm{O}_{2}$-only estimate of energy exchange.

\section{Glucose and Fat Oxidation: Combustion}

The bomb calorimeter provides definitive proof of the energy differences among substrates. So, why is there a difference in heat loss per volume of $\mathrm{O}_{2}$ consumed between glucose and fat? For both oxidative respiration and combustion, the $1.5 \mathrm{~kJ}$ per liter of $\mathrm{O}_{2}$ difference between glucose and fat often is referenced to the efficiency of the fuel. To the contrary, William Thornton's law [2], published in 1917, reveals a rather remarkable similarity in heat loss among various gases undergoing combustion, with heat loss per $\mathrm{O}_{2}$ (and electron) equivalent more closely resembling a single constant in accordance with the energy equivalent of fat not glucose oxidation. In fact, fuel efficiency can differ among oxidized substrates. Variance in heat loss is indicative of the size of the molecules undergoing combustion. Larger molecules are likely to have more proportionate bond types, smaller molecules less, so that greater variability in heat (energy) loss is evident when smaller molecules like glucose are combusted and compared to larger ones like fat [3]. This also is true for respiratory oxidation. But there is more to a series of biochemical reactions as compared to the single oxidative step of combustion.

\section{Glucose and Fat Oxidation: Respiration}

A quick flip through any biochemistry text offers a quite different explanation as to why respiratory glucose oxidation and energy loss would differ from that of fat: anaerobic 
glucose metabolism. Energy expenditure fueled by fat oxidation is entirely represented as mitochondrial $\mathrm{O}_{2}$ uptake. But before the aerobic oxidation of glucose derivatives within mitochondria, energy exchange known as glycolysis takes place that yields ATP yet consumes no oxygen (Figure 1(a)). And ultimately it is our quest to estimate ATP turnover that constitutes our need to quantify energy exchange. It appears, then, that in addition to the energy within the molecule itself, the metabolic processes of how complete respiratory glucose oxidation takes place also need deliberation [4]. In terms of anaerobic and aerobic energy loss, glycolytic ATP production is where respiration and bomb calorimetry (combustion) part ways.

In the 1970s, careful analyses of Gibbs energy $(\Delta G)$, enthalpy $(\Delta \mathrm{H})$, and entropy $(\Delta \mathrm{S})$ changes were completed as glucose was split into 2 molecules of pyruvate or lactate (and 2 net molecules of ATP were resynthesized) [5]. Gibbs energy changes were found to be identical regardless of what the "endproduct" of glycolysis was. With pyruvate as an end product, however, glycolytic Gibbs energy changes were largely accounted for by entropy; with lactate as the end product, Gibbs energy changes were detected by enthalpy (quantified as heat loss). This peculiarity between the two is important because entropy changes cannot be measured; they are instead calculated based on Gibbs energy (product/reactant ratios) and enthalpy measures. Thus, under aerobic conditions (i.e., pyruvate formation), glycolytic energy exchanges are identified by changes that are not typically considered part of an $\mathrm{O}_{2}$ uptake measurement. The $1.5 \mathrm{~kJ}$ difference per liter of $\mathrm{O}_{2}$ between respiratory glucose and fat oxidation acknowledges glycolytic energy exchange regardless of representation by heat or entropy. This theme can be readily applied to substrate utilization and $\mathrm{O}_{2}$ uptake during exercise and recovery.

\section{Glucose, Lactate, and Fat Oxidation: Application}

In addition to his Nobel-winning work disproving muscle as a heat engine (it was instead a chemomechanical converter), A.V. Hill suggested that the volume of the $\mathrm{O}_{2}$ debt incurred after the completion of exercise was affected by the amount of lactate produced and later removed [6]. The idea that lactate levels, in part, dictates the volume of $\mathrm{O}_{2}$ consumed caught on quickly. Note that $\mathrm{O}_{2}$ volumes are being quantified with this description, not energy expenditure based on substrate oxidation. For decades, lactate was thought causal to the $\mathrm{O}_{2}$ debt. Subsequent research however disproved the notion that lactate levels affect $\mathrm{O}_{2}$ consumption in recovery [7]. As a result, in 1984, exercise physiologists suggested a qualitative name change to replace the lactate-associated $\mathrm{O}_{2}$ debt hypothesis: excess postexercise oxygen consumption or EPOC [8].

Keep in mind that while glucose metabolism contains both an anaerobic and aerobic component, lactate oxidation is all aerobic. And the concept being demonstrated is not related to the thermic effect of feeding. Yet, separating glycolytic metabolism from mitochondrial $\mathrm{O}_{2}$ uptake introduces a methodology that recognizes a quantitative difference

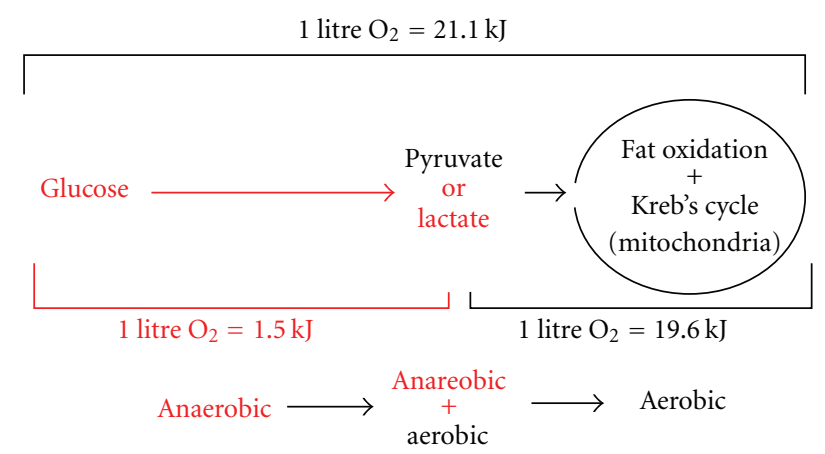

(a)

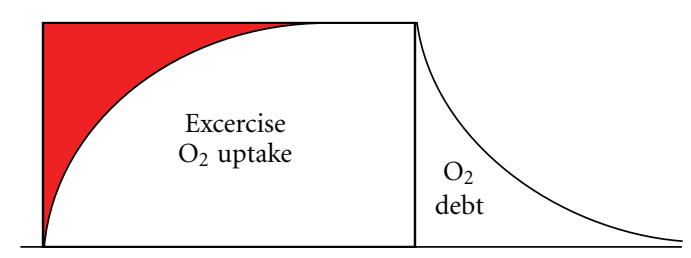

(b)

Figure 1: Anaerobic glycolytic metabolism is depicted in red, aerobic metabolism in black print, from left to right. At the top of the figure, both comprise a respiratory oxygen uptake to energy cost conversion of 1 liter of oxygen $=21.1 \mathrm{~kJ}$ when glucose is used as a fuel. Below, the anaerobic and aerobic components of a brief bout of exercise are shown, followed by an all-aerobic recovery (represented by the term " $\mathrm{O}_{2}$ debt") where fat and lactate are consumed at $19.6 \mathrm{~kJ}$ per liter of oxygen. The $1.5 \mathrm{~kJ}$ per liter of $\mathrm{O}_{2}$ uptake difference is accounted for by entropy changes with pyruvate formation and by enthalpy changes when lactate is produced.

in glucose, fat, and lactate oxidation within cells. With glucose as a fuel, a muscles $\mathrm{O}_{2}$ uptake indeed reflects complete anaerobic plus aerobic glucose oxidation, at 1 liter $\mathrm{O}_{2}$ uptake $=21.1 \mathrm{~kJ}$. Glycolysis with concomitant ATP production can subsequently be dismissed as part of respiratory $\mathrm{O}_{2}$ uptake measure in accordance with Thornton's combustion law, with lactate oxidation being equivalent to fat oxidation where there is no anaerobic component, at 1 liter $\mathrm{O}_{2}$ uptake $=19.6 \mathrm{~kJ}$.

By representing glycolytic ATP resynthesis as a $1.5 \mathrm{~kJ}$ metabolic differential (per $\mathrm{L} \mathrm{O}_{2}$ consumed) between glucose and fat/lactate oxidation, this energy cost component can be dismissed from an $\mathrm{O}_{2}$ debt measurement after the completion of exercise when fat and lactate oxidation are considered prevalent (Figure 1(b)). Does fuel utilization influence the $\mathrm{O}_{2}$ debt? First mentioned in the introduction, one interpretation states that fat oxidation as compared to glucose oxidation will indeed increase the volume of $\mathrm{O}_{2}$ consumed in recovery [9]. Why did not the previously mentioned lactate oxidation $-\mathrm{O}_{2}$ uptake studies promote this rationale? The answer appears to depend on whether the enthalpy and entropy energy exchange components of glycolysis and mitochondrial respiration are or are not considered part of an $\mathrm{O}_{2}$ uptake to energy expenditure estimation. In comparison with glucose, had lactate oxidation been 
expressed as $\mathrm{kJ}$ instead of by liters of $\mathrm{O}_{2}$, it would indeed have some influence on the volume of the $\mathrm{O}_{2}$ debt.

\section{Synopsis}

The concept of entropy, a thermodynamic parameter that is not measured via calorimetry, has been the focus of developmental biology for years [10]. Yet, the nutritional sciences have given little attention to this concept. One immediate application in the recognition of entropy is with the use of $\mathrm{O}_{2}$ uptake measurements to quantify the energy costs of living. In this regard, glucose oxidation has an anaerobic ATP resynthesis component that fat and lactate oxidations do not-this component is not measured with direct calorimetry when pyruvate is formed. Yet, it is there. And it needs to be accounted for if energy expenditure rather than $\mathrm{O}_{2}$ uptake is given consideration.

\section{References}

[1] P. A. Molé and J. J. Hoffmann, "VO 2 kinetics of mild exercise are altered by RER," Journal of Applied Physiology, vol. 87, no. 6, pp. 2097-2106, 1999.

[2] R. M. Thorton, "The relation of oxygen to the heat of combustion of organic compounds," Philosophical Magazine Series, vol. 33, pp. 196-203, 1917.

[3] C. B. Scott and Z. Djurisic, "The metabolic oxidation of glucose: thermodynamic considerations for anaerobic and aerobic energy expanditure," Journal of Exercise Physiology Online, vol. 11, no. 4, pp. 34-43, 2008.

[4] C. B. Scott, A Primer for the Exercise and Nutrition Sciences, Humana Press, Totowa, NJ, USA, 2008.

[5] S. Minakami and C.-H. de Verdier, "Calorimetric study on human erythrocyte glycolysis. Heat production in various metabolic conditions," European Journal of Biochemistry, vol. 65, no. 2, pp. 451-460, 1976.

[6] A. V. Hill, C. N. H. Long, and H. Lupton, "Muscular exercise, lactic acid and the supply and utilization of oxygen," Proceedings of the Royal Society, vol. 96, pp. 438-475, 1924.

[7] D. A. Roth, W. C. Stanley, and G. A. Brooks, "Induced lactacidemia does not affect postexercise $\mathrm{O}_{2}$ consumption," Journal of Applied Physiology, vol. 65, no. 3, pp. 1045-1049, 1988.

[8] G. A. Gaesser and G. A. Brooks, "Metabolic bases of excess post-exercise oxygen consumption: a review," Medicine and Science in Sports and Exercise, vol. 16, no. 1, pp. 29-43, 1984.

[9] R. Bahr, "Excess postexercise oxygen consumption-magnitude, mechanisms and practical implications," Acta Physiologica Scandinavica, Supplement, vol. 144, no. 605, pp. 1-70, 1992.

[10] A. I. Zotin, Thermodynamic Aspects of Developmental Biology, Karger, Basel, Switzerland, 1992. 

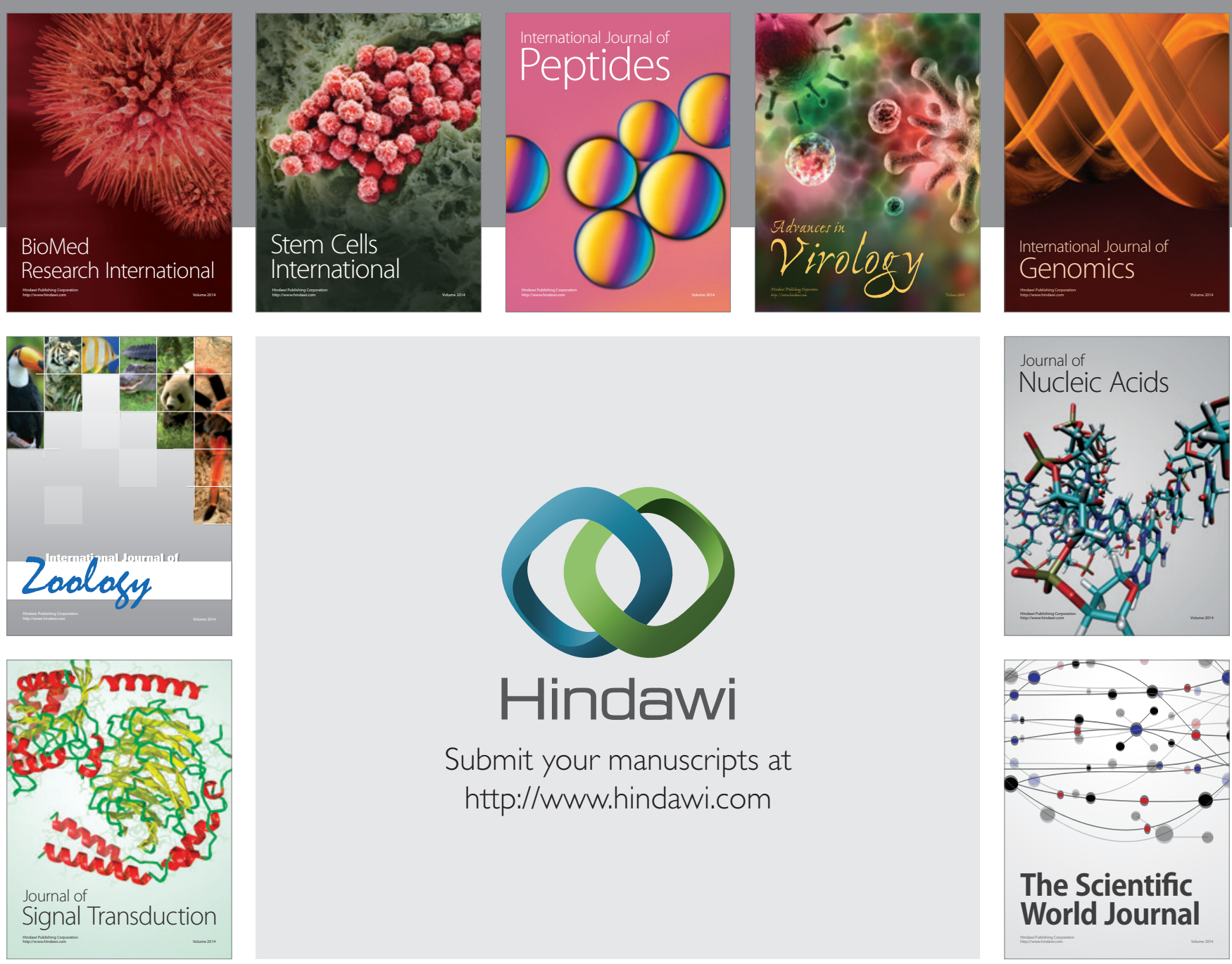

Submit your manuscripts at

http://www.hindawi.com
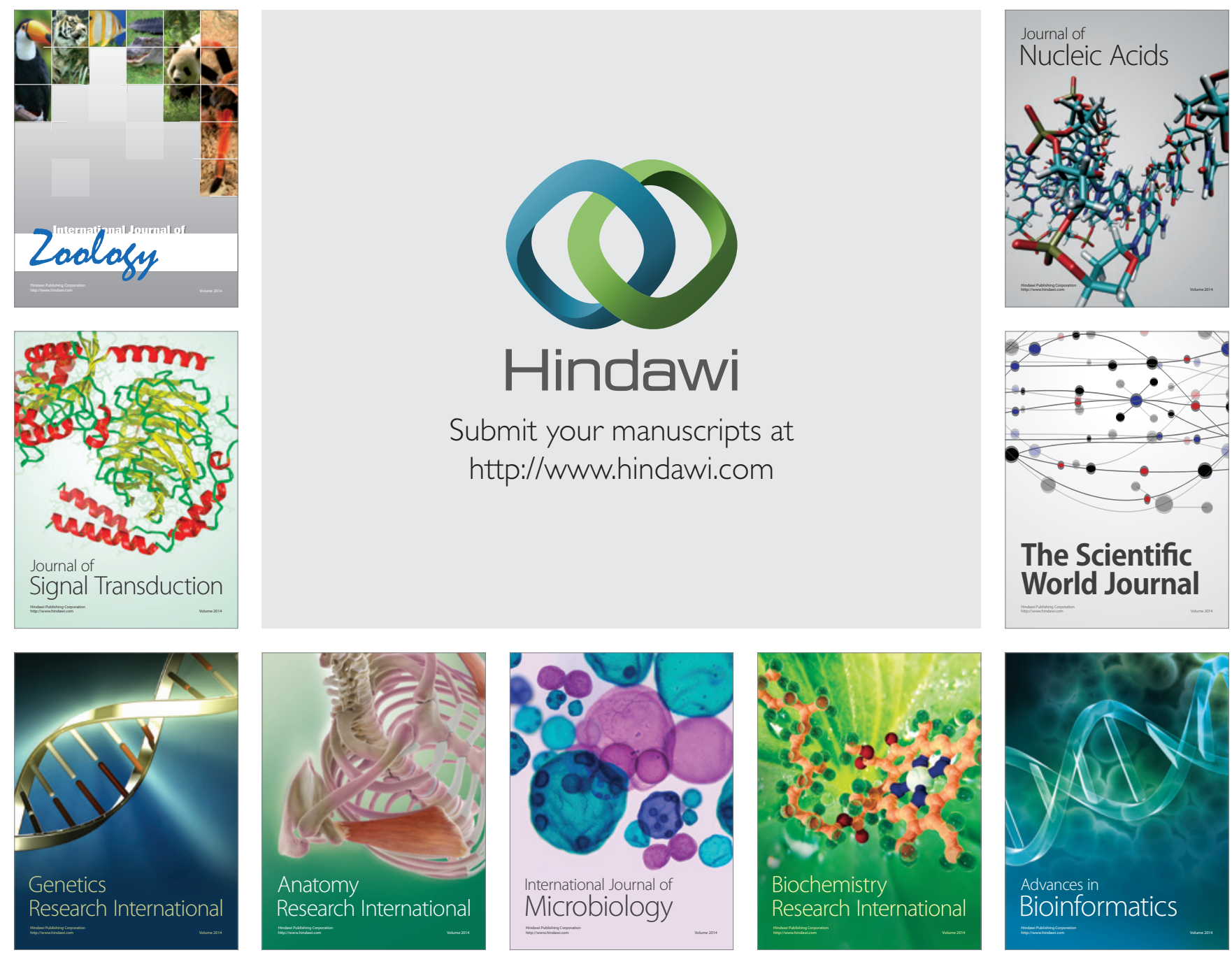

The Scientific World Journal
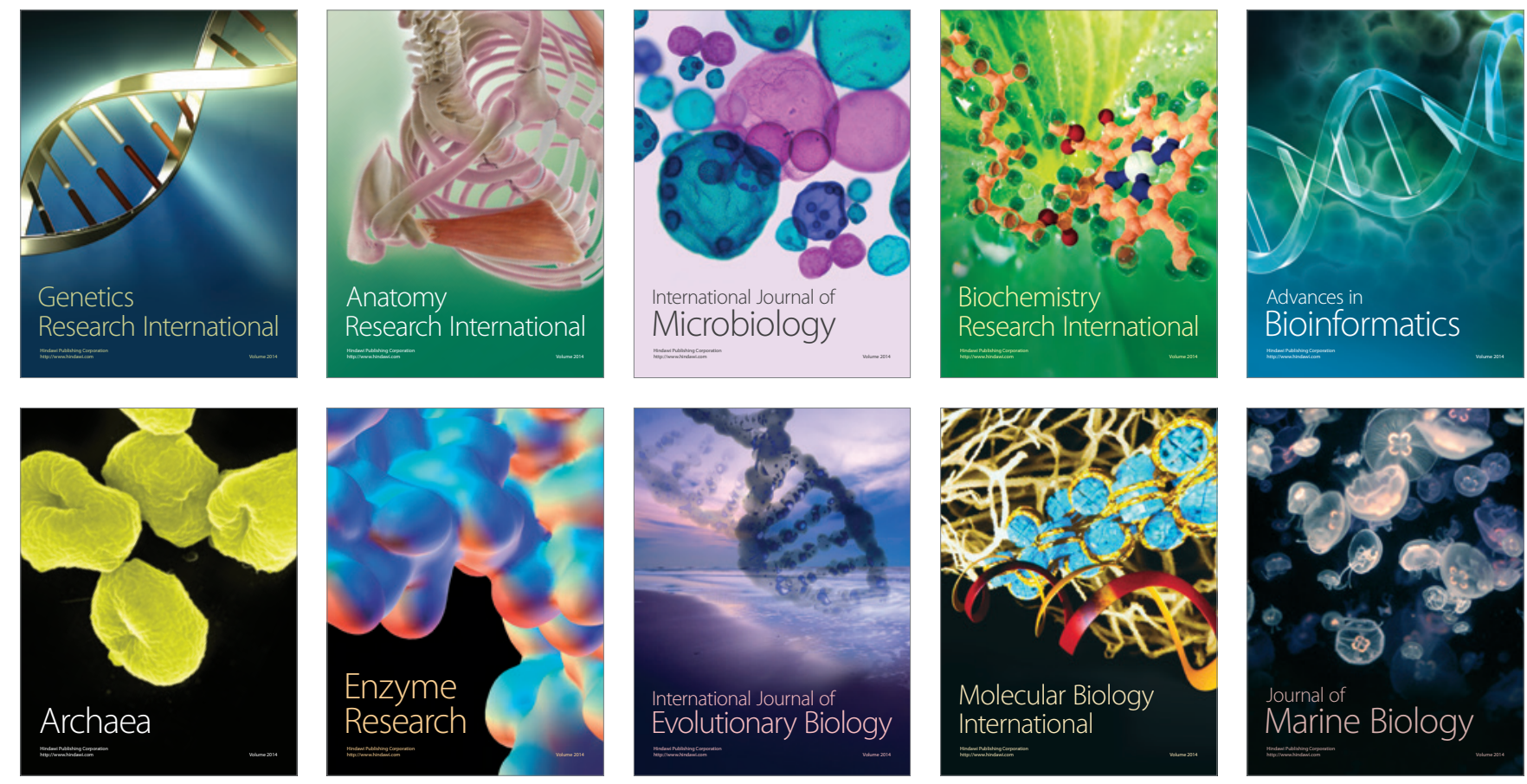